\title{
Retrograde Spread of Buccal Space Infection into Temporal Space with Temporal Muscle Necrosis in a Medically Compromised Patient - A Case Report
}

\author{
Arrvinthan S. U. ${ }^{1}$, Lokesh Bhanumurthy², Jimson Samson ${ }^{3}$, Anandh Balasubramanian ${ }^{4}$ \\ 1,2, 3,4 Department of Oral \& Maxillofacial Surgery, Tagore Dental College \& Hospital, \\ Rathinamangalam, Tamil Nadu, India.
}

\section{INTRODUCTION}

Superficial temporal space lies between the temporal fasciae. Abscess in the temporal and infratemporal space is very rare. They develop as a result of the extraction of infected maxillary molars. Temporal space infections or abscesses can be seen in the superficial or deep temporal regions. A 65 - year - old male patient reported with a complaint of painful swelling over the right cheek and restricted mouth opening with a history of extraction of second mandibular molar before four weeks. On examination, an ill-defined diffuse swelling was seen. Treatment was started with IV empirical antibiotics and planned for surgical drainage. Surgical drainage of the abscess in the temporal space was done along with debridement of the necrosed temporalis muscle.

Infections of the maxillofacial region are of great significance to general dentists and maxillofacial surgeons. They are of clinical importance as they are commonly encountered, and are also challenging as timely intervention is needed to prevent fatal complications. The infections arising from the tooth are initially confined to the alveolar bone and surrounding periosteum. They spread along the path of the least resistance to the cortical plates. Once the infection penetrates the cortical plates, they reach the muscle plane. ${ }^{1}$ If the infection perforated is above the muscle attachments, it's confined to an intraoral abscess. If the cortical plates are perforated below the muscular attachments, extraoral swelling develops. The next barrier is the periosteum which is strong and elastic in nature. Once the periosteum is breached, infections reach the soft tissue planes, the fascia. Most of the infections are confined to a particular space and the surrounding fascia. Based on the toxins produced by the microorganisms, the infection can spread to adjacent spaces and even retrograde. Common deep space infections are Ludwig's angina followed by peritonsillar, submandibular, and parotid abscesses. ${ }^{2}$ Infratemporal and temporal space infections are rarely compared to other deep space infections. Many etiological factors form the base for the infections of deep spaces, dental caries, extraction of infected, non-infected tooth maxillary sinusitis, tonsillitis, maxillary sinus fracture, temporomandibular arthroscopy, drug-induced infections.

Infections of odontogenic origin, spreading along infratemporal and temporal space are most common with maxillary molars followed by mandibular molars. We report a case of retrograde spread of buccal space infection into temporal space secondary to mandibular tooth extraction.
Corresponding Author: Dr. Jimson Samson,

Professor \& Head of the Department, Tagore Dental College \& Hospital, Rathinamangalam, Melakottaiyur (PO), Chennai- 600127, Tamil Nadu, India.

E-mail:maxfacs@tagoredch.in

DOI: $10.14260 / j e m d s / 2021 / 669$

How to Cite This Article:

Arrvinthan SU, Bhanumurthy L, Samson J, et al. Retrograde spread of buccal space infection into temporal space with temporal muscle necrosis in a medically compromised patient - a case report. $J$ Evolution Med Dent Sci 2021;10(37): 3301-3305, DOI:

10.14260/jemds/2021/669

Submission 29-06-2021,

Peer Review 24-08-2021,

Acceptance 30-08-2021,

Published 13-09-2021.

Copyright (C) 2021 Arrvinthan S.U. et al. This is an open access article distributed under Creative Commons Attribution License [Attribution 4.0 International (CC BY 4.0)] 


\section{PRESENTATION OF CASE}

- A 65 - year - old male came to our Department of Oral and Maxillofacial Surgery OPD, with the chief complaint of difficulty in mouth opening and pain and swelling in the lower back tooth region for the past 15 days. History of presenting illness revealed, the pain was a radiating type of pain to the right temporal region and aggravated on mastication and swelling in the right cheek region for the past 15 days. Past dental history revealed traumatized extraction of 48 under local anaesthesia before 1 month elsewhere.

- Medical history revealed, the patient was under medication for renal disorder but stopped the medication without any consultation with his treating physician. Personal history revealed the patient was a chronic alcoholic and smoker for the past 20 years. He had quit the habit of smoking before 2 years.

- On general examination, the patient was conscious, oriented, and febrile. On local examination, restricted mouth opening with an interincisal distance of less than $20 \mathrm{~mm}$ (Figure 1). Moderate swelling over the right side of the cheek and right temporal region was present (Figure 2). On palpation, acute tenderness over the 48 region, pterygomandibular region and the right temporal regions were present.

- Investigations proceeded with USG. USG report revealed $2.9 \times 1.7 \mathrm{~cm}$ echoic soft tissue swelling just anterior to the ramus of the mandible in the subcutaneous plane with peripheral vascularity and surrounding soft tissue oedema on the right side. Treatment was planned for incision and drainage under local anaesthesia.

During his stay in the hospital, the patient was admitted to the OMFS ward, complete blood investigations, renal function test (RFT), liver function test (LFT), hepatitis B and C, HIV tests were done. Investigations revealed elevated WBC count and urea, creatinine values. Nephrologists' opinion was obtained. An IV line was started with empirical antibiotic therapy of taxim $1 \mathrm{~g} \mathrm{IV}$, metrogyl $500 \mathrm{mg}$ IV along with analgesics.

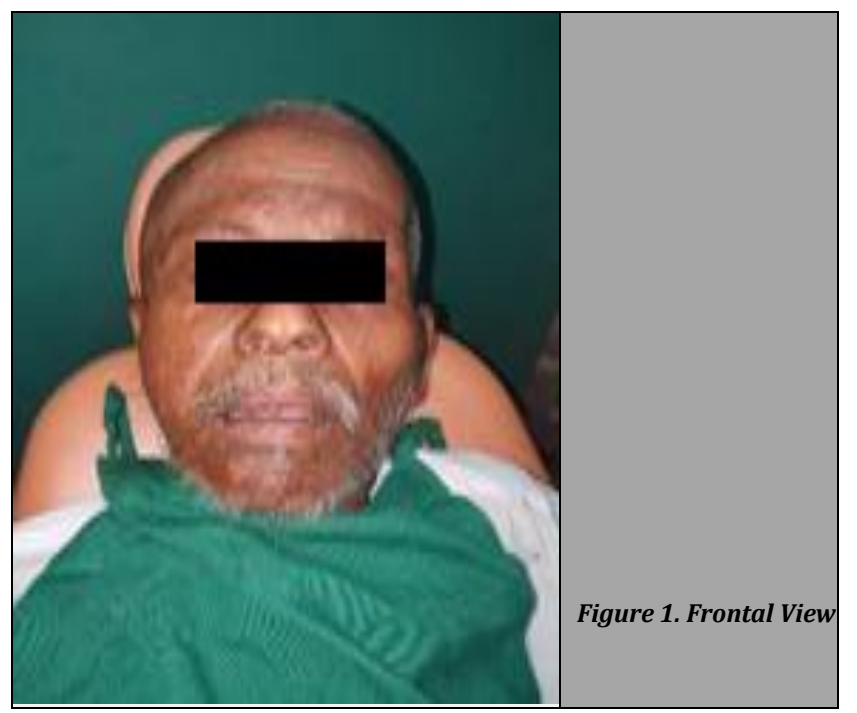

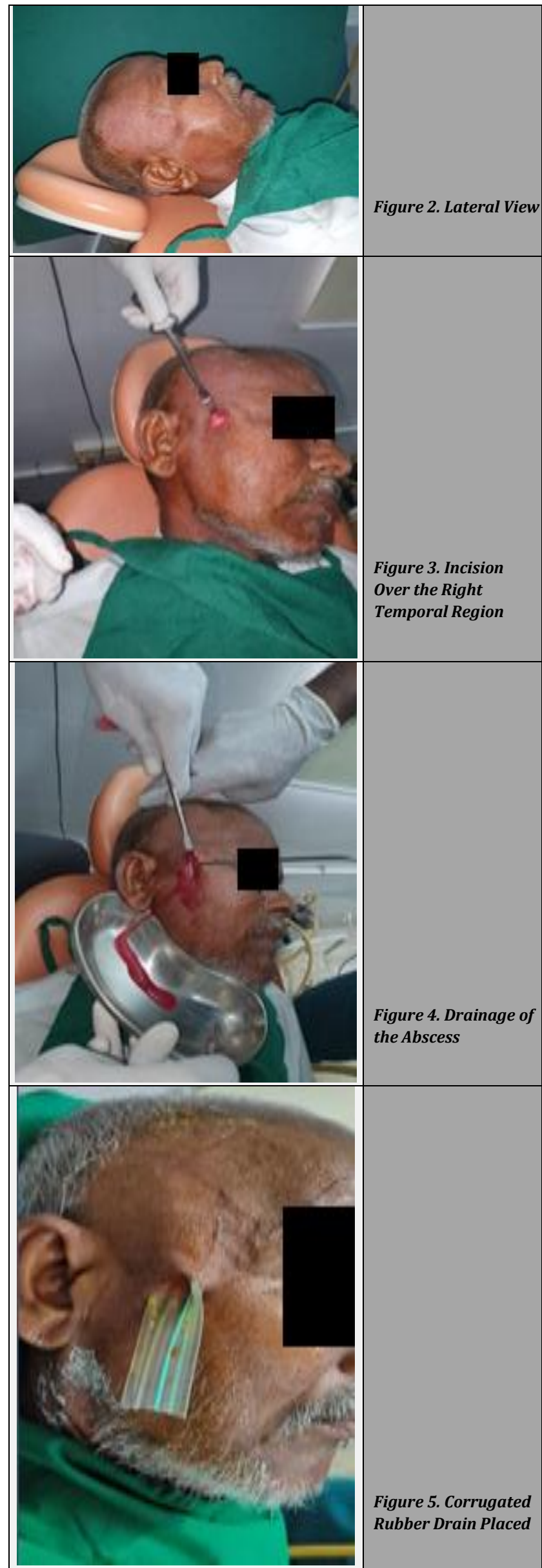




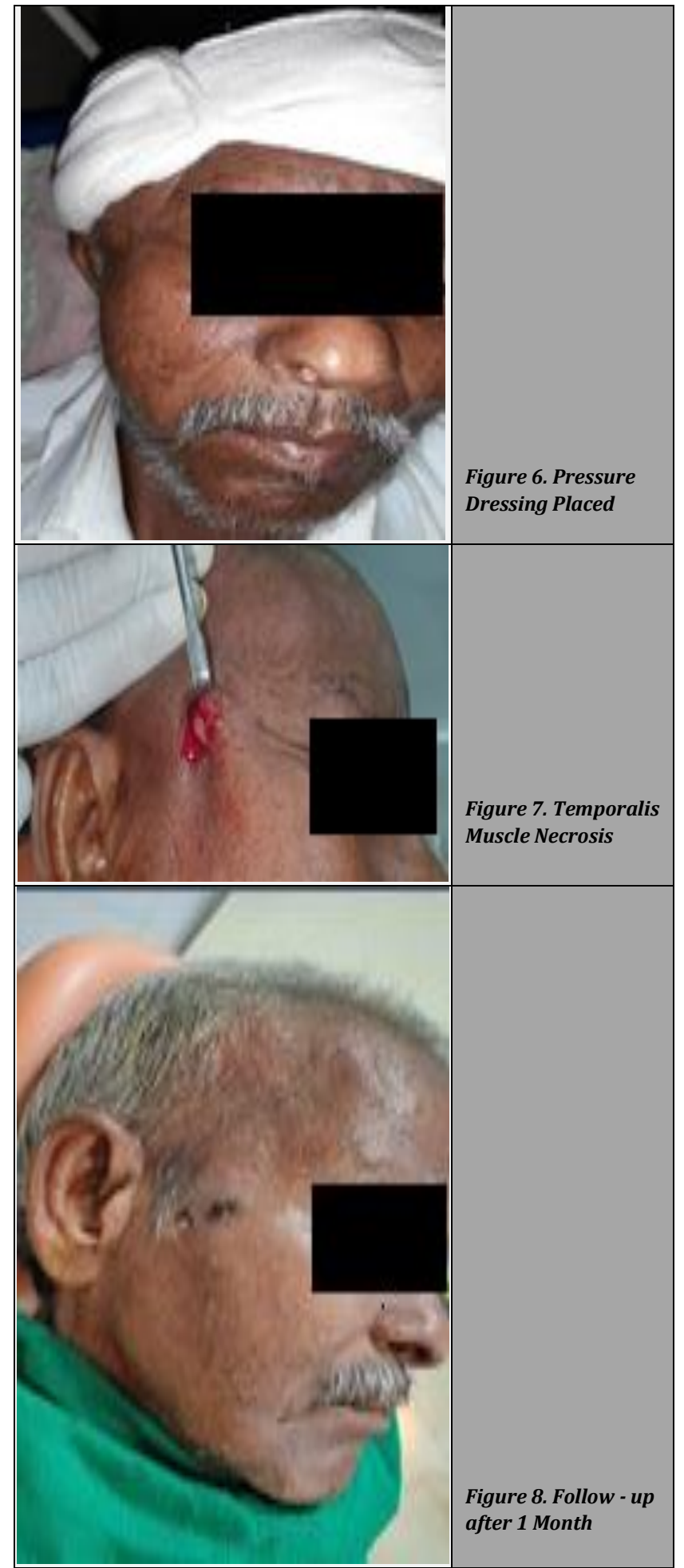

\section{Surgical Procedure}

Patient was placed in a semi - supine position. Painting of the surgical site with betadine done. 1:80,000 lignocaine with adrenaline was administered in the surgical site as right inferior alveolar nerve block along with infiltration given in the right temporal region.

We planned an extraoral incision and drainage to drain the swelling over the right temporal region. An incision was placed (Figure 3) and dissection was done with curved haemostat and abscess drained (Figure 4), with further dissection we could dissect out the necrosed fibres of temporalis muscle (Figure 7). The wound was washed with betadine and metrogyl several times; corrugated rubber drain was placed and secured in position with sutures (Figure 5).

An intraoral stab incision was placed over the pterygomandibular raphe in relation to 48 region, dissection was done with curved haemostat and abscess drained, washed with betadine and metrogyl and a corrugated rubber drain was placed and secured in position with suture. The pressure dressing was done in the temporal region (Figure 6). The intraoral and extraoral abscesses were collected separately and sent for culture sensitivity. Followed up with constant irrigation both intraorally and extraorally.

The culture and sensitivity report revealed Pseudomonas aeruginosa gram-negative bacilli. The antibiotic was changed to cefoperazone + sulbactam 1.2 gm IV along with metrogyl $500 \mathrm{mg}$ IV along with analgesics. Renal function test (RFT) report revealed urea level as $90 \mathrm{mg} / \mathrm{dl}$, creatinine $6.6 \mathrm{mg} /$ dl. The patient was again referred to the nephrologist and an opinion was obtained.

After the first 3 days post-op, intraoral and extraoral discharge from the drains were collected and again sent for antibiotic culture sensitivity, which revealed klebsiella, resistant with cefoperazone + sulbactam, hence antibiotics were changed to piptaz 1.5 gm IV. The patient was under constant renal function monitoring. After 8 days post-op, the extraoral drain ceased and the drain was removed and sutured with 4 - 0 ethilon. The patient was discharged with oral antibiotic therapy for the next 5 days.

\section{DISCUSSION}

Odontogenic infections are the most common forms of head and neck infections. ${ }^{3}$ Infections from the tooth spread through the surrounding fascial spaces after breaching the periosteum. Infections from the mandibular molars commonly spread to either of the spaces or in combination with the following spaces, the sublingual, submandibular, buccal, submasseteric, pterygomandibular space or the lateral pharyngeal space. ${ }^{4}$ The superficial temporal pouch / space easily communicates with the submasseteric space and the deep temporal space or pouch communicates with the infratemporal space, lateral pharyngeal space, ${ }^{1,4}$ pterygomandibular space. They are divided into superficial and deep temporal spaces. The superficial temporal space extends superiorly to the pericranium, lateral to the temporalis muscle and medial to the temporoparietal fascia, inferiorly it's continuous with the masseteric space. Deep temporal space extends superiorly to the attachment of the temporalis muscle to the inferior temporal crest and lateral to the temporal bone and deep to the temporalis muscle, this space is continuous inferiorly with the infratemporal space. They contain a buccal fat pad and three veins and the infections from the buccal space spread along with the buccal fat pad to the superficial temporal fascia. ${ }^{4}$ Temporal space abscess is a rare type of space infection, with an incidence of $0.74 \% 5$ reported in the literature. Necrotizing soft tissue infections may be bacterial or fungal in origin. These types of necrotizing infections occur around $8.9 \%$ in the head and neck region. In our case, we had 
encountered necrosis of the temporalis muscle which is rare to see in head and neck space infections. While dissecting through the incised area in the temporal region, we could see the necrosed muscle fibres. Several etiological factors may have influenced the cause for the necrosis including age, comorbidities, and personal habits like alcoholism. ${ }^{7}$

The clinical features can be from moderate swelling to severe swelling in the temporal region. The characteristic feature of the temporal space infection is Trismus, which is due to the spasm of the temporalis muscle. ${ }^{8}$ Bratton et al. stated that for diagnosing head and neck space infections, CT is standard than MRI. ${ }^{9}$ Most common pathogens include gram-positive aerobic alphahaemolytic streptococci, 4,10 facultative anaerobes within the Streptococcus anginosus group and gram-negative rods like prevotella, porphyromonas and fusobacterium species. ${ }^{10}$

Assessment of the airway and management is important in the management of deep head and neck space infections. ${ }^{11}$ Surgical drainage remains the mainstay treatment for the management of head and neck space infections. Whenever surgical drainage is necessary Topazian and Goldberg recommended the following principles: incise in healthy skin and mucosa when possible, not at the site of maximum fluctuance, because these wounds tend to heal with an unsightly scar; place the incision in a natural skin fold; place the incision in a dependent position; dissect bluntly; place a drain; and remove drains when drainage becomes minimal. ${ }^{12}$ In cases where there is necrosis of soft tissues, surgical debridement is necessary to prevent further complications.

Management of the temporal space infection can be done with extraoral incision placed in the temporal region, above the hairline and the incision being angled at 45 degrees to the zygomatic arch. ${ }^{5}$ Blunt dissection of the fascia is done with a haemostat. Facial nerve branches, especially the zygomatic and temporal branches of the facial nerve should be kept in mind while dissecting through the fascia. If the infection is confined to the superficial temporal space or pouch, the dissection is confined only to temporal fascia. If the infection is in the deep temporal space or pouch, then dissection through the temporalis muscle should be done to drain the abscess. The healing of the abscessed cavity takes place by fibrosis and there will be persistent trismus postoperatively and physiotherapy must be advised to prevent the muscle fibrosis and post-operative trismus. In our case, in addition to incision \& drainage of the abscess, we had to treat the temporalis muscle necrosis, with careful surgical debridement, so that we could prevent the inadvertent exposure of the underlying structures and prevent injury to the branches of the facial nerve with repeated antibiotic dressings in the area of necrosis, and we could prevent the further spread of necrosis.

Post-operative antimicrobial therapy will play a key role in the drained abscess region. In situations where the patients have comorbidities, patients with chronic kidney disease as in our case, the use of antimicrobial resistance and the need for usage of higher antibiotics are challenging. In this case, we had to manage the resistance to common antibiotics and had to start higher antibiotics based on the culture sensitivity report, with constant monitoring of the renal function to prevent furthermore complications. Piperacillin / tazobactam combination is effective in most resistant cases and polymicrobial involvement. ${ }^{13}$ Initially, our patient had sensitivity to cefoperazone / sulbactam combination, later with subsequent abscess collection sent for culture sensitivity, we found to have resistance, so we changed the antibiotic therapy to Piperacillin / tazobactam combination.

Medically compromised situations, present with a challenge to equally manage the systemic health status and local presenting conditions in a balanced manner without causing a flare-up of their systemic conditions. Patients with chronic kidney diseases, patients having poor oral hygiene, and a need for dental prophylaxis are always in association with other systemic conditions such as hypertension and diabetes mellitus. They are generally immunocompromised and are highly prone to odontogenic infections. The effects of renal failure often are widespread and can involve multiple systems - more than $40 \%$ of patients also have diabetes, and more than $15 \%$ have concurrent hypertension. ${ }^{14}$ Comorbidities might be also a factor for soft tissue necrosis, this might have been one of the factors for temporalis muscle necrosis in our case.

\section{CONCLUSIONS}

Temporal space abscess can occur secondary to masseteric space infection. According to our case report, drainage of abscess from temporal space and infratemporal space by intraoral approach and if the infection persists then drain the abscess by extraoral approach to drain the pus from remaining masticator spaces. All the masticator spaces can communicate with surrounding spaces, in the spread and drainage of abscesses. Management of space infections in medically compromised patients is always challenging to treat the source of infection without flaring up the systemic conditions. Muscle necrosis is rare in infections of odontogenic origin, but it should also be kept in mind while treating such infections.

Financial or other competing interests: None.

Disclosure forms provided by the authors are available with the full text of this article at jemds.com.

\section{REFERENCES}

[1] Borle R, Angik R. Odontogenic infections. Textbook of Oral \& Maxillofacial Surgery. $1^{\text {st }}$ edn. Jaypee Publication 2014: p. 329.

[2] Narayana ML, Gaur U, Chaithanya RN, et al. A rare case of temporal and infratemporal space abscess secondary to messeteric space infection. International Journal of Otorhinolaryngology and Head and Neck Surgery 2020;6(2):384.

[3] Uluibau IC, Jaunay T, Goss AN. Severe odontogenic infections. Australian Dental Journal 2005;50(Suppl 4):S74-81. 
[4] Osborn TM, Assael LA, Bell RB. Deep space neck infection: principles of surgical management. Oral Maxillofac Surg Clin North Am 2008;20(3):353-65.

[5] Peterson LJ. Peterson's Principles of Oral and Maxillofacial Surgery. USA: People's Medical Publishing House 2012: p. 2000.

[6] Gujrathi AB, Ambulgekar V, Kathait P. Deep neck space infection - a retrospective study of 270 cases at tertiary care center. World Journal of Otorhinolaryngology Head and Neck Surgery 2016;2(4):208-13.

[7] Headley AJ. Necrotizing soft tissue infections: a primary care review. Am Fam Physician 2003;68(2):323-8.

[8] Fonseca RJ, Walker RV. Oral and maxillofacial trauma. Philadelphia: WB Saunders 1991: p. 1360.

[9] Bratton TA, Jackson DC, Nkungula-Howlett T, et al. Management of complex multi - space odontogenic infections. J Tenn Dent Assoc 2002;82(3):39-47.
[10] Stefanopoulos PK, Kolokotronis AE. The clinical significance of anaerobic bacteria in acute orofacial odontogenic infections. Oral Surg Oral Med Oral Pathol Oral Radiol Endod 2004;98(4):398-408.

[11] Cho YS, Woo HJ, Kim JY, et al. Airway management in patients with deep neck infections: a retrospective analysis. Medicine (Baltimore) 2016;95(27):e4125. Erratum: Medicine (Baltimore) 2016;95(42):e36c2.

[12] Topazian RG, Goldberg MH. Oral and Maxillofacial Infections. W.B. Saunders Company, 1994: p. 653.

[13] Gorbach SL. Piperacillin / tazobactam in the treatment of polymicrobial infections. Intensive Care Medicine 1994;20(Suppl 3):S27-34.

[14] Little JW, Falace DA, Miller CS, et al. Chronic kidney disease and dialysis. Little and Falace's Dental management of the medically compromised patient. Elsevier 2013: p. 186-99. 